\title{
Noise Diagnostics of Scooter Faults by Using MPEG-7 Audio Features and Intelligent Classification Techniques
}

\author{
Mingsian R. Bai*, Meng-Chun Chen and Jian-Da Wu
}

Department of Mechanical Engineering, National Chiao-Tung University, 1001 Ta-Hsueh Road, Hsin-Chu 300, Taiwan

\begin{abstract}
A scooter fault diagnostic system that makes use of feature extraction and intelligent classification algorithms is presented in this paper. Sound features based on MPEG (Moving Picture Experts Group)-7 coding standard and several other features in the time and frequency domains are extracted from noise data and preprocessed prior to classification. Classification algorithms including the Nearest Neighbor Rule (NNR), the Artificial Neural Networks (ANN), the Fuzzy Neural Networks (FNN), and the Hidden Markov Models (HMM) are employed to identify and classify scooter noise. A training phase is required to establish a feature space template, followed by a test phase in which the audio features of the test data are calculated and matched to the feature space template. The proposed techniques were applied to classify noise data due to various kinds of scooter fault, such as belt damage, pulley damage, etc. The results reveal that the performance of methods is satisfactory, while varying slightly in performance with the algorithm and the type of noise used in the tests.
\end{abstract}

\section{INTRODUCTION}

Taiwan is one of the major scooter manufacturers in the world. Fault diagnosis is an important element in the manufacturing and maintenance process of scooters. In the past, the diagnosis of scooter fault generally relied on well-trained technicians. However, this experience-oriented approach is not only inefficient but also prone to human errors and inconsistencies. It is highly desirable to identify and classify scooter faults in a systematic, automatic and reliable fashion, using machines instead of humans. To this end, this paper proposes a noise diagnostic system on the basis of sound features and intelligent classification techniques for scooter faults.

Fault diagnostics for machines can be largely divided into two categories: the model-based methods and the signalbased methods. For example, Bai et al. [1] developed an online fault detection and isolation technique for the diagnosis of rotating machinery. The system consists of a signal-based feature generation module and a model-based fault inference module. Bai et al. [2] also proposed an order tracking system for the diagnosis of rotating machinery. The recursive least squares (RLS) algorithm and the Kalman filter are exploited to extract the order amplitudes of vibration signals as features, followed by fault classification using the fuzzy state inference module. On the other hand, Jia et al. [3] reported a noise diagnosis technique based on wavelet and fuzzy Cclusters for connecting rod bearing fault. The system is capable of classifying four kinds of bearing fault. Wavelet packet proved to be robust to background noise while extracting fault characteristics. Wang et al. [4] suggested a method to diagnose misfire of internal combustion engines based on singular value decomposition (SVD) and artificial neural networks (ANN).

*Address correspondence to this author at the Department of Mechanical Engineering, National Chiao-Tung University, 1001 Ta-Hsueh Road, HsinChu 300, Taiwan; E-mail: msbai@mail.nctu.edu.tw
Extraction of features is an important step prior to a classification system. Since we are mainly dealing with noises due to various types of scooter faults, sound features are derived from the measured noises. In this research, the sound features are based on the MPEG (Moving Picture Experts Group)-7 standard [5]. Although MPEG-7 was originally intended for audio signals, we found it useful to use 11 of the MPEG-7 descriptors for describing noise content. Slightly different in purpose, Crysandt [6] used MPEG-7 descriptors to classify music of multimedia contents. Peeters [7] conducted a comprehensive survey on audio features for sound description in the Content-based Unified Interfaces and Descriptors for Audio/music Databases available Online (CUIDADO) project. Some of the noise descriptors in this research are also derived from his work. Nineteen auditory features including Audio Spectrum Centroid, Linear Predictive Coding (LPC) adopted in this work are summarized in APPENDIX.

Given the abundant set of sound features, one needs to choose only features that are most effective to the problem at hand. There are ways for one to select features according to the nature of problem. Xavier et al. [8-10] suggested several pre-selection techniques including Discriminant Analysis (DA) [8], Mutual Information (MI), and Gradual Descriptor Elimination (GDE) [9-10]. Zongker et al. [11] evaluated the quality of feature subsets and compared their computational requirements. The Sequential Forward Selection (SFS) algorithm was found to be quite effective for selecting features.

There are also plenty of choices for classification techniques. In the present work, the Nearest Neighbor Rule (NNR) [12], the Artificial Neural Network (ANN) [13, 14], the Fuzzy Neural Network (FNN) [15-17], and the Hidden Markov Model (HMM) [18-20] were employed to classify the noises due to scooter faults. These methods have been extensively used in a variety of problems such as music classification [14], continuous Mandarin word recognition [19], and so forth. These methods achieve high detection rates by forming highly nonlinear decision boundaries in the feature 


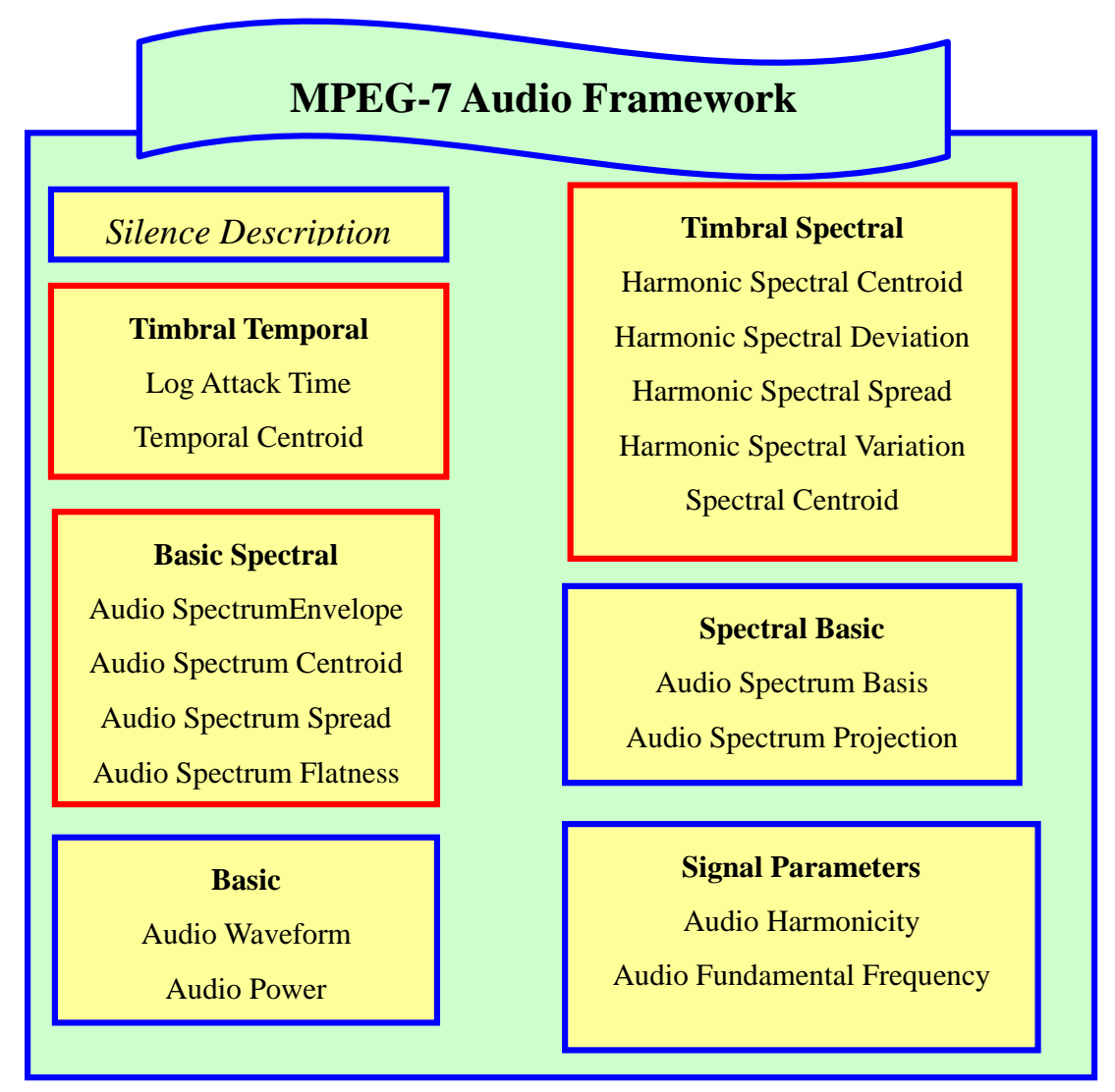

Fig. (1). The MPEG-7 audio framework. The audio framework consists of seventeen low level descriptors of temporal and spectral audio features that can be divided into six groups.

space. Each of these algorithms will be reviewed in the following sections in more detail. The overall architecture of the proposed diagnostic system is depicted in Fig. (2). Experiments were conducted for noise data measured on scooters to validate the proposed intelligent diagnostic system. Fault types such as belt damage and pulley damage were examined.

\section{FEATURE EXTRACTION}

Sound features generally fall into three categories: spectral features, temporal features and statistical features. Nineteen features are used in this study (see APPENDIX). The majority of the sound features are taken from MPEG-7 [5] which is an ISO/IEC standard for describing the multimedia content. Seventeen descriptors in MPEG-7 can be categorized into six groups: Timbral Temporal, Timbral Spectral, Basic Spectral, Basic, Signal Parameters, and Spectral Basis, as shown in Fig. (1). Only the first three groups are used in this research. Among the Timbral Temporal descriptors, the Log Attack Time (LAT) characterizes the attack of a sound, or the time it takes for the signal to rise from silence to the maximum amplitude. It signifies the difference between a sharply changed sound and a smoothly changed sound.

LAT $=\log _{10}\left(T_{1}-T_{0}\right)$,

where $T_{0}$ is the time when the signal starts and $T_{1}$ is the time when it reaches its maximum.
The Temporal Centroid (TC) characterizes the signal envelope, signifying where in time the energy of a signal concentrates. It is defined as follows:

$$
\mathrm{TC}=\frac{\sum_{n=1}^{\text {length }(\mathrm{SE})} \frac{n}{\mathrm{SR}} \cdot \mathrm{SE}(n)}{\sum_{n=1}^{\text {length }(\mathrm{SE})} \mathrm{SE}(n)},
$$

where $\operatorname{SE}(n)$ is the signal envelope at time instant $n$, calculated using the Hilbert Transform [23] and SR is the sampling rate.

The Basic Spectral descriptors are obtained from the time-frequency analysis of the audio signal. The Audio Spectrum Envelope (ASE) describes the short-term power spectrum of an audio signal as a time-series of spectra on a logarithmic frequency scale in $1 / 4$ octave resolution. It may be used to display a spectrogram, and is defined as follows:

$$
\operatorname{ASE}(k)=\frac{|A(k)|^{2}}{l w \cdot N F F T} \quad k=\frac{1}{16}, \frac{1}{8}, \frac{1}{4}, \frac{1}{2}, 1,2,4,8 \mathrm{kHz}
$$

where NFFT is the FFT size, $l w$ is the window length, $A(k)$ is the magnitude of a component in the frequency range, 62.5 $\mathrm{Hz}$ and $8 \mathrm{kHz}$.

The Audio Spectrum Centroid (ASC) calculates the center of gravity of the log-frequency power spectrum. It indi- 

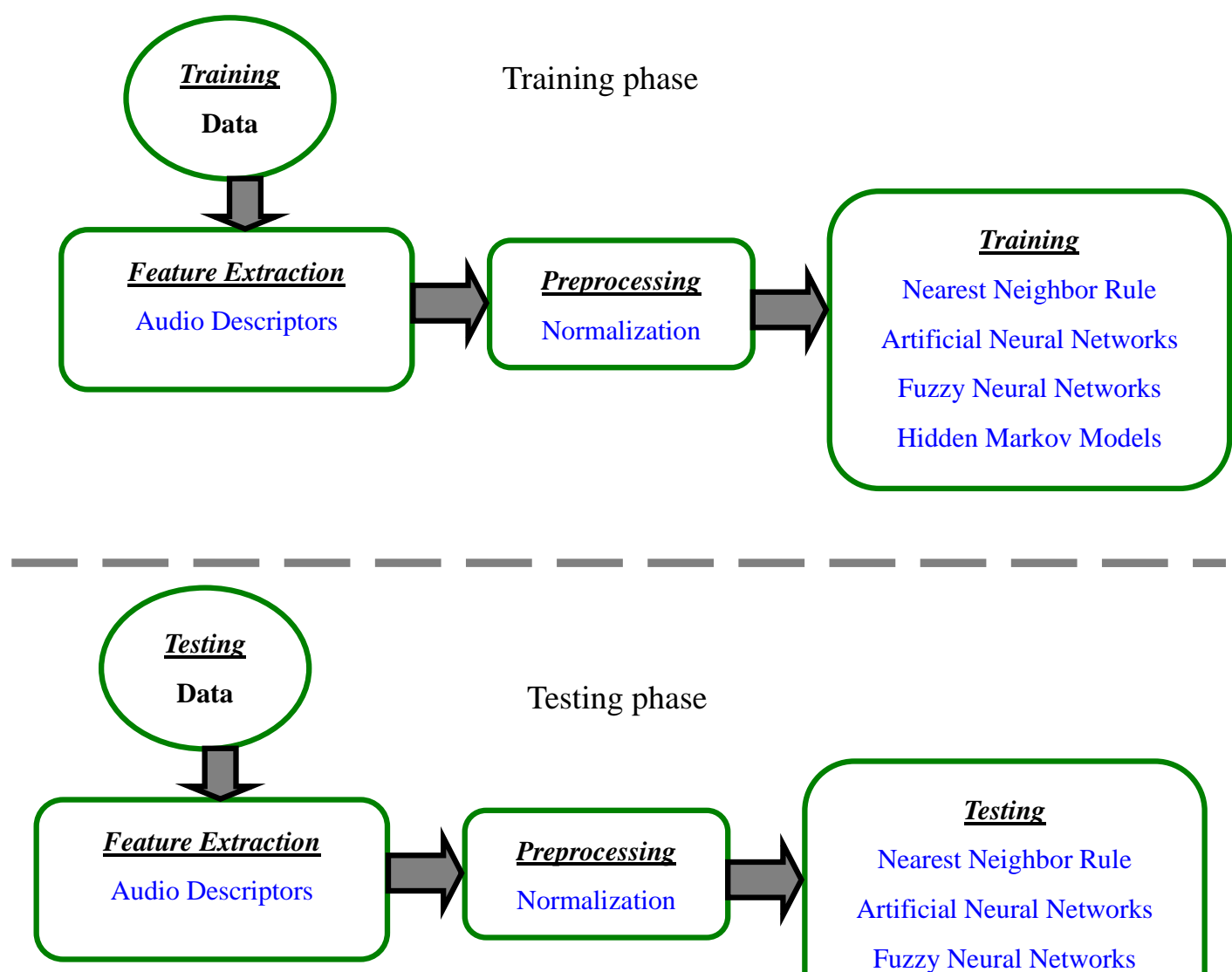

cates whether the spectral content of the signal dominates in high or low frequencies.

$$
\mathrm{ASC}=\frac{\sum_{k} \log _{2}\left(\frac{f(k)}{1000}\right) P(k)}{\sum_{k} P(k)},
$$

where $P(k)$ is the power spectrum magnitude associated with the frequency $f(k)$ in $\mathrm{Hz}$.

The Audio Spectrum Spread (ASS) is the second moment of the log-frequency power spectrum, indicating the spread of the power spectrum with respect to the spectral centroid.

$$
\mathrm{ASS}=\sqrt{\frac{\sum_{k}\left(\left(\log _{2}\left(\frac{f(k)}{1000}\right)-\mathrm{ASC}\right)^{2} P(k)\right)}{\sum_{k} P(k)}}
$$

The Audio Spectrum Flatness (ASF) signifies the flatness of the signal spectrum.

$$
\mathrm{ASF}=\frac{\sqrt[k h(b)-k l(b)+1+1]{\prod_{k=k l(b)}^{k h(b)} P(k)}}{\frac{1}{k h(b)-k l(b)+1} \sum_{k=k l(b)}^{k h(b)} P(k)},
$$

where $k l(b)$ and $k h(b)$ are the lower and higher edges of the band $b$, respectively. An ASF value much greater than unity indicates the presence of tonal components.

The Timbral Spectral descriptors describe the spectral characteristics of sounds in a linear-frequency scale. The Spectral Centroid (SC) is the power-weighted average of the frequency bins in the linear power spectrum.

$\mathrm{SC}=\frac{\sum_{i=1}^{N_{f}} \operatorname{ISC}(i)}{N_{f}}$,

where 


$$
\operatorname{ISC}(i)=\frac{\sum_{k=1}^{\text {length }(S)} f_{i}(k) P_{i}(k)}{\sum_{h=1}^{\text {length }(S)} P_{i}(k)},
$$

where $N_{f}$ is the number of frames and ISC $(i)$ is the instantaneous spectral centroid of the $i$ th frame

The Harmonic Spectral Centroid (HSC) is the amplitudeweighted mean of the harmonic peaks of a spectrum.

$\mathrm{HSC}=\frac{\sum_{i=1}^{N_{f}} \operatorname{IHSC}(i)}{N_{f}}$,

where

$$
\operatorname{IHSC}(i)=\frac{\sum_{h=1}^{N_{h}} f_{i}(h) A_{i}(h)}{\sum_{h=1}^{N_{h}} A_{i}(h)},
$$

where $N_{h}$ is the number of harmonic peaks, $i$ is the frame index, $f_{i}(h)$ is the frequency in $\mathrm{Hz}$ of the $h$ th harmonic, $A_{i}(h)$ is the magnitude of the $h$ th harmonic, and $\operatorname{IHSC}(i)$ is the instantaneous harmonic spectral centroid of the $i$ th frame.

The Harmonic Spectral Deviation (HSD) indicates the spectral deviation of the log-amplitude components from a global spectral envelope.

$$
\begin{aligned}
& \operatorname{HSD}=\frac{\sum_{i=1}^{N_{f}} \operatorname{IHSD}(i)}{N_{f}}, \\
& \operatorname{IHSD}(i)=\frac{\sum_{h=1}^{N_{h}}\left|\log _{10}\left(A_{i}(h)\right)-\log _{10}\left(S E_{i}(h)\right)\right|}{\sum_{h=1}^{N_{h}} \log _{10}\left(A_{i}(h)\right)}, \\
& S E_{i}(h)= \begin{cases}\frac{A_{i}(h)+A_{i}(h+1)}{2} & \text { when } h=1 \\
\frac{\sum_{l=-1} A_{i}(h+l)}{3} & \text { when } h \in\left[2, N_{h}-1\right] \\
\frac{A_{i}(h-1)+A_{i}(h)}{2} & \text { when } h=N_{h}\end{cases}
\end{aligned}
$$

where $S E_{i}(h)$ is the $h$ th harmonic spectral envelope and $\operatorname{IHSD}(i)$ is the instantaneous harmonic spectral deviation of the $i$ th frame.

The Harmonic Spectral Spread (HSS) is the amplitudeweighted standard deviation of the harmonic peaks of the spectrum, normalized by the instantaneous Harmonic Spectral Centroid (IHSC).
$\mathrm{HSS}=\frac{\sum_{i=1}^{N_{f}} \operatorname{IHSS}(i)}{N_{f}}$,

$$
\operatorname{IHSS}(i)=\frac{1}{\operatorname{IHSC}(i)} \sqrt{\frac{\sum_{h=1}^{N_{h}} A_{i}^{2}(h) \cdot\left(f_{i}(h)-\operatorname{IHSC}(i)\right)^{2}}{\sum_{h=1}^{N_{h}} A_{i}^{2}(h)}},
$$

where $\operatorname{IHSS}(i)$ is the instantaneous harmonic spectral spread of the $i$ th frame and $\operatorname{IHSC}(i)$ is defined in Eq.(10).

The Harmonic Spectral Variation (HSV) is the normalized correlation between the amplitudes of the harmonic peaks of two adjacent frames.

$$
\begin{gathered}
\mathrm{HSV}=\frac{\sum_{i=2}^{N_{f}} \operatorname{IHSV}(i)}{N_{f}-1}, \\
\operatorname{IHSV}(i)=1-\frac{\sum_{h=1}^{N_{h}} A_{i-1}(h) \cdot A_{i}(h)}{\sqrt{\sum_{h=1}^{N_{h}} A_{i-1}{ }^{2}(h)} \sqrt{\sum_{h=1}^{N_{h}} A_{i}^{2}(h)}},
\end{gathered}
$$

where $A_{i-1}(h)$ represents the magnitude of the $h$ th harmonic peak of the previous frame, and IHSV(i) is the instantaneous harmonic spectral variation of the $i$ th frame.

In addition to the MPEG-7 descriptors, we also use other types of feature including the LPC and MFCC. LPC [21] derives the coefficients of a forward linear predictor using the Levinson-Durbin recursion algorithm:

$$
\hat{x}(n)=-a(2) x(n-1)-a(3) x(n-2)-\cdots-a(p+1) x(n-p),
$$

where $\hat{x}(n)$ is the estimated signal, $p$ is the order of the prediction filter, $a(2), \cdots, a(p+1)$ are the coefficients of the predictor, and $x(n)$ is the input signal.

MFCC [22] are cepstrum coefficients derived from the discrete cosine transform (DCT) based on the critical bandwidths of human hearing. These critical bandwidths are $100 \mathrm{~Hz}$ below $1 \mathrm{kHz}$, but rise nearly exponentially above 1 $\mathrm{kHz}$. Human perception of sound can be regarded as a nonuniform filter bank, with fine resolution at low frequencies and coarse resolution at high frequencies, arranged according to the Mel-scale frequency scale. The relationship between the Mel-scale frequency and linear frequency is given by

$$
m e l_{-} f(k)=2595 * \log _{10}\left(1+\frac{f(k)}{700}\right) \text {, }
$$

where $\mathrm{mel}_{-} f(k)$ is the Mel-scale frequency and $f(k)$ is the linear frequency. The computation of MFCC starts with calculating the energy $E(m)$ of the $m$ th band 


$$
E(m)=10 \cdot \log \left[\sum_{k}|X(k)|^{2} B_{m}(k)\right],
$$

where $X(k)$ is the FFT of the signal $x(n), B_{m}(k)$ is the triangular weighting function [22] of the $m$ th band and the summation is taken only for the frequency components in the $m$ th band. After that, the energy $E(m)$ is converted to the Quefrency domain by using discrete cosine transform (DCT) to result in the Mel-scale cepstrum coefficients:

$\operatorname{MFCC}(n)=\frac{1}{M} \sum_{m=1}^{20} \cos \left[\frac{\pi n(m-0.5)}{M}\right] E(m)$

Finally, Sound Pressure Level (SPL) in dB is also employed as one of the sound features in the work. Zerocrossing rate measures the rate of sign-changes in a signal. Pitch represents the fundamental frequency of the spectrum. Statistical descriptors including Autocorrelation, Skewness and Kurtosis are also included in the feature set. Skewness is a measure of asymmetry of data about the mean and is defined as

$S K=\frac{E\left\{(x-\mu)^{3}\right\}}{\sigma^{3}}$,

where $E\{\}$ denotes the expectation operation, $\mu$ and $\sigma$ are the mean and the standard deviation, respectively, of the time-domain data $x$. Kurtosis is a measure of how outlierprone a distribution is. The kurtosis $K$ of a random variable $x$ is defined as

$$
K=\frac{E\left\{(x-\mu)^{4}\right\}}{\sigma^{4}} \text {. }
$$

In feature extraction, normalization is usually necessary to ensure numerical performance of the ensuing classification process. The features calculated using the aforementioned procedures are normalized as follows:

Step 1. Divide the features into several parts according to the extraction methods.

Step 2. Find the minimum and the maximum in each data set.

Step 3. Rescale each data so that the maximum of each data is 1 and the minimum of each data is -1 . That is, the features fall into the full range within the interval $[-1,1]$.

In order to simplify the processing, it is usually desirable to reduce the feature set to its minimum. To this end, the following Sequential Forward Selection (SFS) procedure can be used:

Step 1. Find the single feature that yields the highest successful detection rate.

Step 2. Keep the already-selected features and try to identify a newly added feature that yields the highest successful detection rate.
Step 3. Repeat step 2 until the desired number of features has been selected or until there is no further improvement in the successful detection rate.

\section{CLASSIFICATION METHODS}

In this section, a review of several algorithms used for the present noise classification problem is given. Only the concepts relevant to the current discussion are addressed.

\section{A. Nearest Neighbor Rule}

NNR [12] is a straightforward approach to the problem of classification. In this method, we simply find the closest object from the training set. The thus found object is associated with the same class as that training data. The Euclidean distance is used as the distance measure:

$\operatorname{dist}\left(x, x_{\text {train }}\right)=\left|x-x_{\text {train }}\right|^{2}$,

where $x_{\text {train }}$ is the training data and $x$ is the object being classified. The flowchart of NNR is shown in Fig. (3).

\section{B. Artificial Neural Networks}

ANN [13] is an important technique in artificial intelligence that mimics human's learning process. Biological learning involves adjustments to the synaptic connections that exist between the neurons, as shown in Fig. (4). The relationship of the input and output of a neuron can be represented by the following equation:

$y=f(n e t)=f\left(\sum_{n=1}^{N_{x}} w_{n} x_{n}+\theta\right)$,

where $y$ is the output, $x_{n}$ is the input, $w_{n}$ is the weighting function, $N_{x}$ is the number of input nodes, $\theta$ is the bias, and $f(\bullet)$ is the activation function which is usually a nonlinear function. In this paper, the following hyperbolic tangent function is used:

$f(x)=\tanh (x)$.

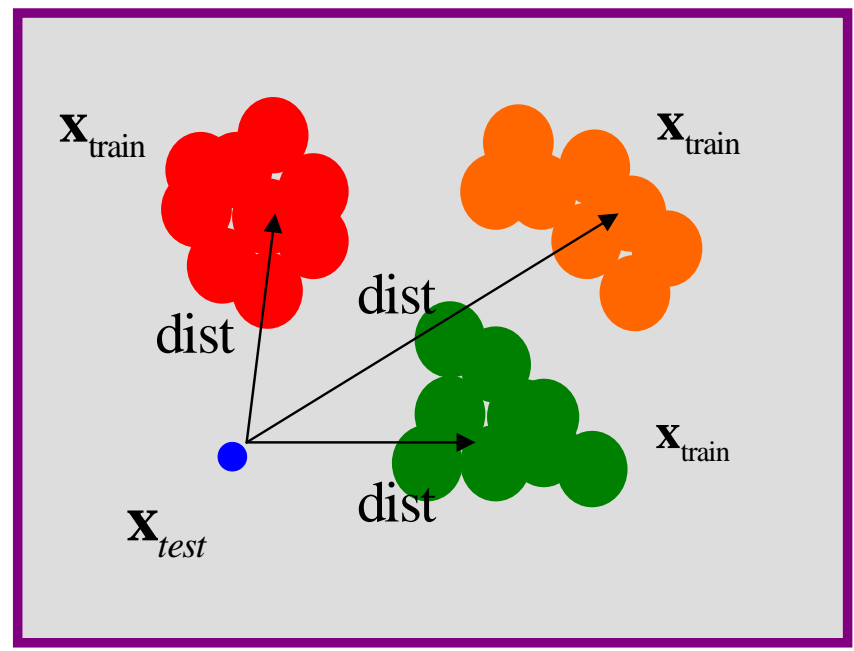

Fig. (3). The idea underlying the NNR algorithm. The distance measure is the Euclidean norm. 


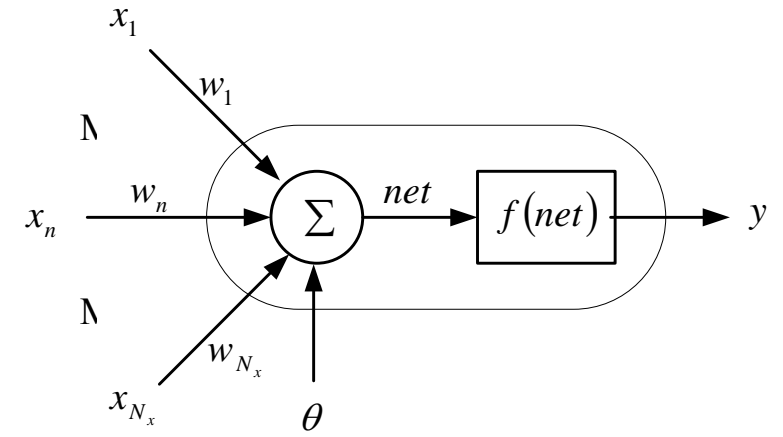

Fig. (4). The schematic structure of a neuron. Learning process in ANN involves adjustments of weights assigned to the connections between the neurons.

Multilayer feedforward networks are widely used which can be shown as Fig. (5). Extending the above input and output relationship of the neuron to the multilayer feedforward network leads to the following expression:

$y_{o}=f_{o}\left[\sum_{h=1}^{N_{z}} w_{o h} \cdot g_{h}\left(\sum_{i=1}^{N_{x}} v_{h i} x_{i}+\theta_{v h}\right)+\theta_{w o}\right]$,

where $x_{i}$ is the input to the input layer, $y_{o}$ is the output from the output layer, $v_{h i}$ is the weighting function between the input layer and the hidden layer, $w_{\text {oh }}$ is the weighting function between the hidden layer and the output layer, $N_{x}$ is the number of neurons in the input layer, $N_{Z}$ is the number of neurons in the hidden layer, $N_{y}$ is the number of neurons in the output layer, $\theta_{v h}$ is the bias of the hidden layer, $\theta_{w o}$ is the bias of the output layer, $g_{h}(\bullet)$ is the activation function of the hidden layer, and $f_{o}(\bullet)$ is the activation function of the output layer.

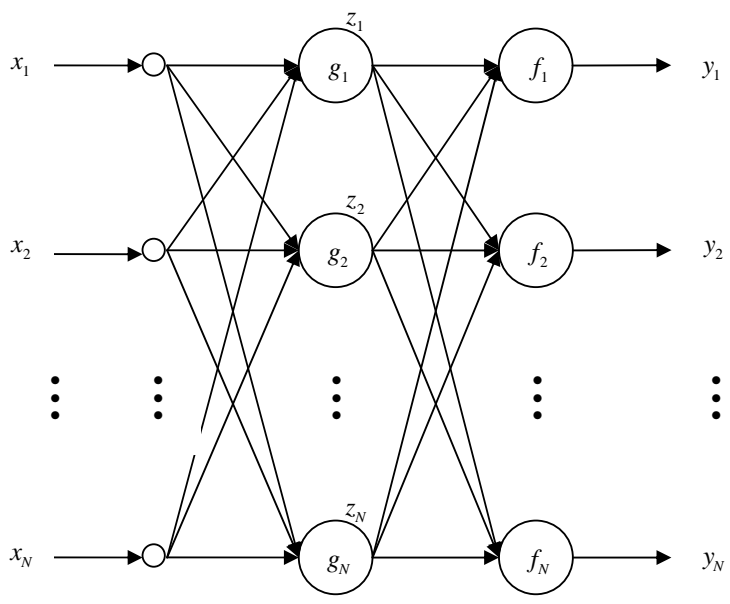

Fig. (5). The structure of a multilayer feedforward ANN. The ANN used in this paper has three layers including the input layer, the hidden layer and the output layer.
The features of signals are used as the inputs to the network. While training the network, the outputs are compared to the targets of the training set. The error between the outputs and the targets is "back propagated" to the network and updates the weightings functions of the nodes in the hidden and output layers. The procedure of back propagation algorithm is given as follows:

Step 1. Decide the network structure and number of neurons.

Step 2. Initialize the network weighting functions.

Step 3. Provide input targets of training set.

Step 4. Calculate the network outputs.

Step 5. Calculate the cost function based on the current weighting functions.

Step 6. Update the weighting functions by using the gradient descent method.

Step 7. Repeat step 3 to step 6 until the network converges.

\section{Fuzzy Neural Networks}

FNN [17] is a technique that combines fuzzy reasoning with ANN. With reference to Fig. (6), the FNN adopted in this paper has five layers: the input layer, the membership layer, the rule layer, the hidden layer, and the output layer.

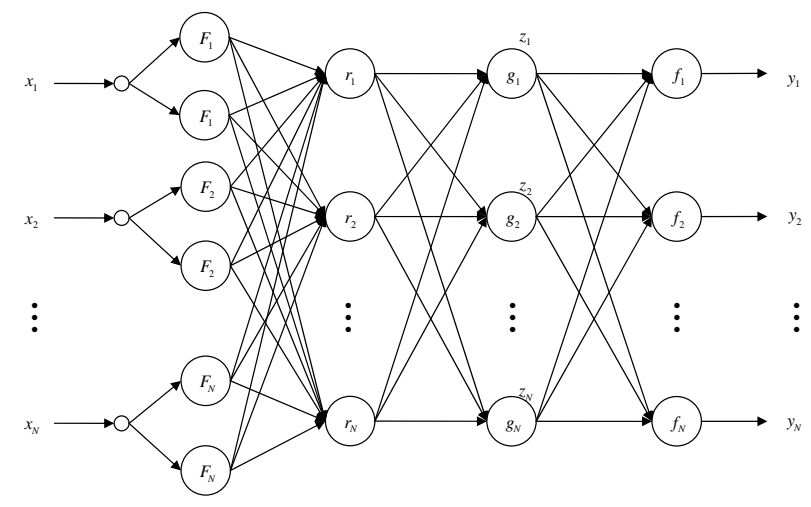

Fig. (6). The structure of the FNN. The FNN used in this paper has five layers including the input layer, the membership layer, the rule layer, the hidden layer and the output layer.

The features of signals are used as the inputs to the network. The second layer is the membership function that connects the fuzzy logics with the neural networks. In the paper, the Gaussian function is selected as the membership function:

$$
F_{i}=e^{\frac{-\left(x_{i}-\mu_{0}\right)^{2}}{2 \sigma_{0}^{2}}},
$$

where $\mu_{0}$ and $\sigma_{0}$ are the mean and the standard deviation, respectively, of the Gaussian function, and $x$ is the feature of the signal. The fuzzy reasoning rule is established with the following format: 
If $x_{1}$ is $F_{1}$ and ... and $x_{N x}$ is $F_{N x}$ then $y_{1}$ is $w_{11}$ and ... and

$y_{N y}$ is $w_{N y N z}$,

where $x_{1}, \ldots, x_{N x}$ are the features of the signal, $F_{1}, \ldots, F_{N x}$ are the associated membership functions, $y_{1}, \ldots, y_{N y}$ are the outputs of the FNN, and $w_{11}, \ldots, w_{N y N z}$ are the weights of the neural network.

The third layer is the rule layer and can be expressed as follows:

$$
r=\prod F_{i}
$$

where $r$ is the truth value of the rule.

The third layer of the FNN is the input layer of the neural network. We use the preceding ANN to train the FNN. The Centroid method is used to obtain the output of the FNN as follows:

$$
y_{o}=f_{o}\left[\sum_{h=1}^{N_{z}} w_{o h} \cdot g_{h}\left(\sum_{i=1}^{N_{x}} v_{h i} r_{i}+\theta_{v h}\right)+\theta_{w o}\right]
$$

\section{Hidden Markov Models}

HMM [18] is a very powerful technique for modeling the speech signals and therefore is widely used in speech recognition. An HMM is characterized by five parameters. The first and the second parameters are the number of states in the model $(N)$ and the number of distinct observation symbols per state $(M)$. The third parameter is the state transition probability distribution ( A ):

$$
\left\{\begin{array}{l}
\mathbf{A}=\left\{a_{i j}\right\} \\
a_{i j}=P\left(q_{t+1}=S_{j} \mid q_{t}=S_{i}\right), \quad 1 \leq i, j \leq N
\end{array},\right.
$$

where $S=\left\{S_{1}, S_{2}, \ldots, S_{N}\right\}$ denotes the set of the states and $q_{t}$ denotes the state at time $t$.

The fourth one is the observation symbol probability distribution ( B ):

$$
\left\{\begin{array}{ll}
\mathbf{B}=\left\{b_{j}(k)\right\} & \\
b_{j}(k)=P\left(V_{k} \text { at } t \mid q_{t}=S_{j}\right), & 1 \leq j \leq N \\
& 1 \leq k \leq M
\end{array} .\right.
$$

where $V=\left\{V_{1}, V_{2}, \ldots, V_{M}\right\}$ denotes the set of the symbols.

The fifth one is the initial state distribution $(\pi)$ :

$$
\left\{\begin{array}{l}
\pi=\left\{\pi_{i}\right\} \\
\pi_{i}=P\left(q_{1}=S_{i}\right), \quad 1 \leq i \leq N
\end{array} .\right.
$$

An HMM is in general denoted as $\lambda=(A, B, \pi)$ for convenience. Three fundamental problems for HMM design can be stated as follows [18]:

Problem 1. Given the observation sequence

$$
O=o_{1}, o_{2}, \cdots, o_{T} \text { and a model } \lambda=(A, B, \pi),
$$

how do we efficiently compute $P(O \mid \lambda)$, the probability of the observation sequence?

Problem 2. Given the observation sequence $O=o_{1}, o_{2}, \cdots, o_{T}$ and a model $\lambda=(A, B, \pi)$, how do we choose an optimal state sequence $Q=q_{1}, q_{2}, \cdots, q_{T}$ that best explains the observations?

Problem 3: How do we adjust the model parameters $\lambda=(A, B, \pi)$ to maximize the probability of the observation sequence $P(O \mid \lambda)$ ?

The solution of the problem 1 can be obtained by using a forward-backward procedure. The solution of the problem 2 can be obtained by using the Viterbi algorithm. The solution of the problem 3 can be obtained by using the Baum-Welch algorithm. The details of these HMM solution algorithms can be found in Reference [18].

In this paper, we use HMM to classify noises due to isolated scooter faults. The block diagram of an HMM-based classifier is shown in Fig. (7). It is assumed that we have $V$ types of noise to classify and each type is modeled by a distinct HMM. The observations are sound features extracted from the noises corresponding to each fault type. The states are variations in spectral composition of the noise.

Step 1. For each fault type $V$, we construct a model $\lambda^{v}$ by estimating the model parameters that optimize the likelihood of observation vectors in the training set.

Step 2. For each unknown fault type to be classified, calculate the features of the noise data and form the observation sequence $O=\left\{\begin{array}{llll}o_{1} & o_{2} & \cdots & o_{T}\end{array}\right\}$. Compute the likelihoods for all possible models $P\left(O \mid \lambda^{v}\right), \quad 1 \leq v \leq V$ by solving problems 1 and 2 using the Viterbi algorithm. Select the model that yields maximum likelihood.

$v^{*}=\arg \max _{1 \leq v \leq V}\left[P\left(O \mid \lambda^{v}\right)\right]$.

\section{EXPERIMENTAL INVESTIGATION}

In order to validate the proposed noise classification techniques, a series of experiments are performed for scooters. Various noise types were created purposely on the platform of an engine rig, as shown in Fig. (8). A scooter with an electronic fuel injection system, single-cylinder, four-stroke, 0.125 -liter internal combustion engine was employed in this application. A 1/2-inch condenser microphone was used to measure the noise produced by the scooter. The experiments were undertaken in a semi-anechoic room. Three practical cases were investigated in the experiments.

Case 1. Three kinds of noise including a muffler expansion noise, a one way clutch noise, and a belt damage noise were examined. These noises were measured while the engine was running. The spectrogram of each noise data is shown in Fig. (9a-c). 


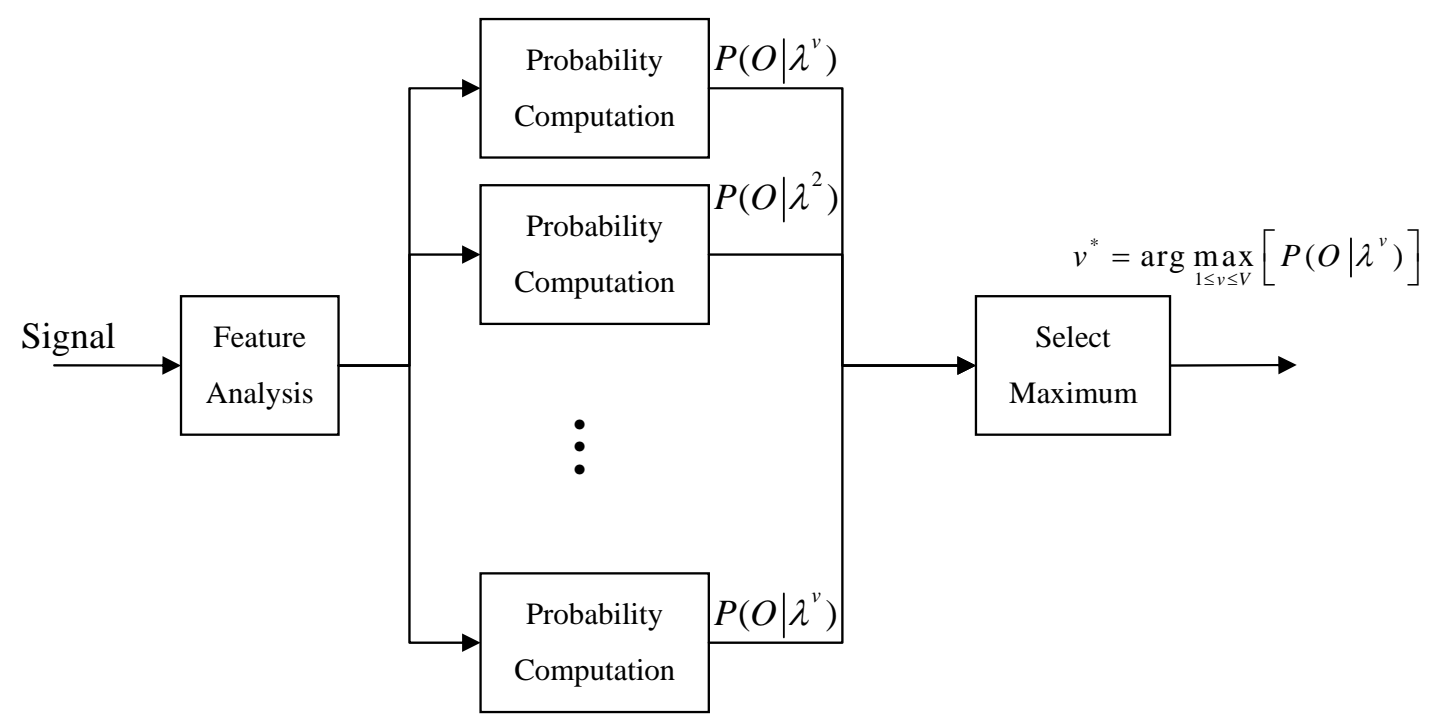

Fig. (7). The block diagram of an HMM-based classifier. In the paper, the computation of probability $P\left(O \mid \lambda^{v}\right)$ is carried out by using the Viterbi algorithm.

The time-domain waveform of each noise data is shown in Fig. (10a-c). The associated features are calculated and shown in Fig. (11).

Case 2. Seven kinds of noise including an ac generator noise, a one way clutch noise, a noise of the engine R-cover, a knocking noise of the engine Lcover, a knocking noise of the engine R-cover, a knocking noise of the engine back-cover, and a knocking noise of the engine top-cover were examined. The first three kinds of noise were measured while the engine was running, whereas the others were measured while the engine is idle.

Case 3. Five kinds of noise including the sound from the engine under the normal condition, the noise due to intake manifold leakage, the clutch damage noise, the pulley damage noise, and the belt damage noise were examined. The noises were measured while the engine was running.

In the experiments, noise data were measured and stored under the sampling rate $44.1 \mathrm{KHz}$ with 16 bit resolution. In a total, 600 frames were employed in each case, where $5 / 6$ of the data was used for training, while $1 / 6$ for testing. The signal processing parameters are: hop size $=441$, window $/$ frame size $=1323$, and FFT size $=2048$.

Feature extraction and noise classification were then performed according to the procedure is depicted in Fig. (2). Features of the noise were extracted and normalized into the range $[-1,1]$. The dimension of the feature space template was 75 . With the input features and the target fault types, we started to train the diagnostic system. The previously mentioned classification techniques, NNR, ANN, FNN, and HMM were applied to the scooter noise data. After training, we entered the testing phase to identify the noise types.

The performance of the classification methods is compared in terms of the successful detection rate which is

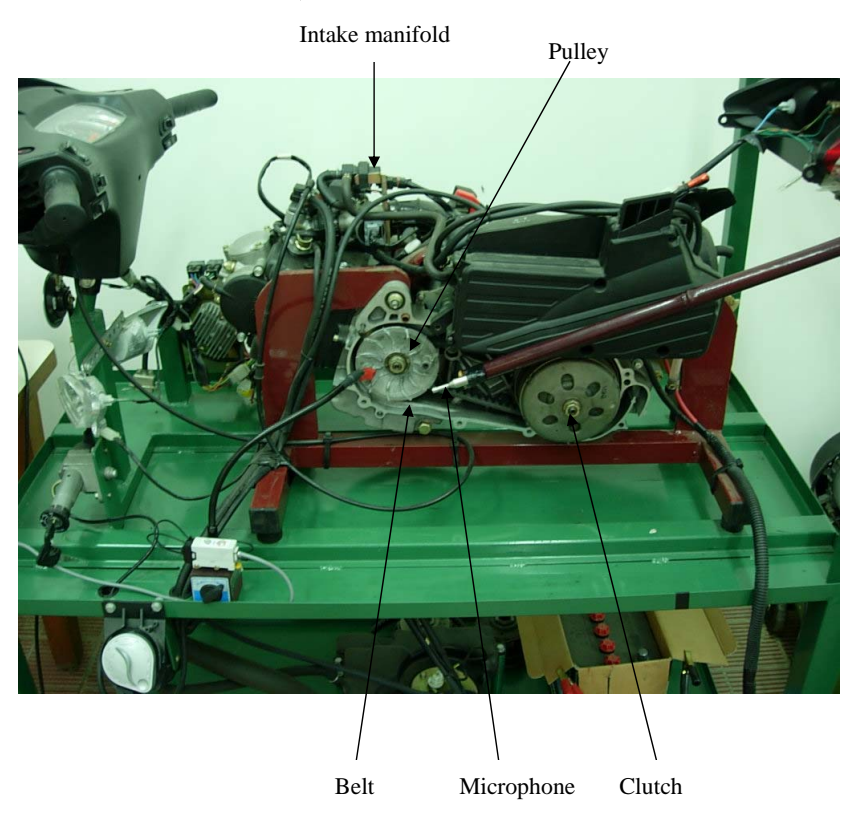

Fig. (8). The Photo of the scooter test rig for the experiments on which various modes of faults can be created.

defined as the ratio of the number of successful detection and the total number of the test frames. For clarity, Tables 1-3 summarize the experimental results of cases 1-3, respectively. The dimension of the feature space is 75 . The number of training data is 500 frames and the number of testing data is 100 frames. The performance of classification methods are compared in terms of successful detection rate which is the ratio of the number of frames of correct identification and the total number of frames. The successful detection rate of case 1 was all very high $(100 \%)$ because the difference of the three noises was clearly audible, even with human hearing. For a more difficult situation of case 2 , the successful detection rate was still high (from $86 \%$ of NNR to $90 \%$ of HMM), but slightly varying with different fault types. Among which, 
(a)

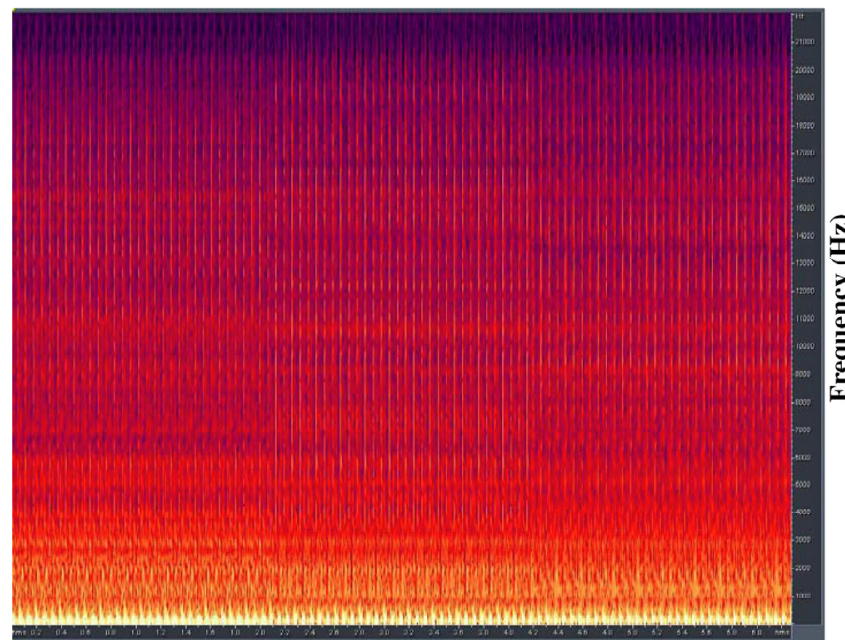

Time (sec)

(b)

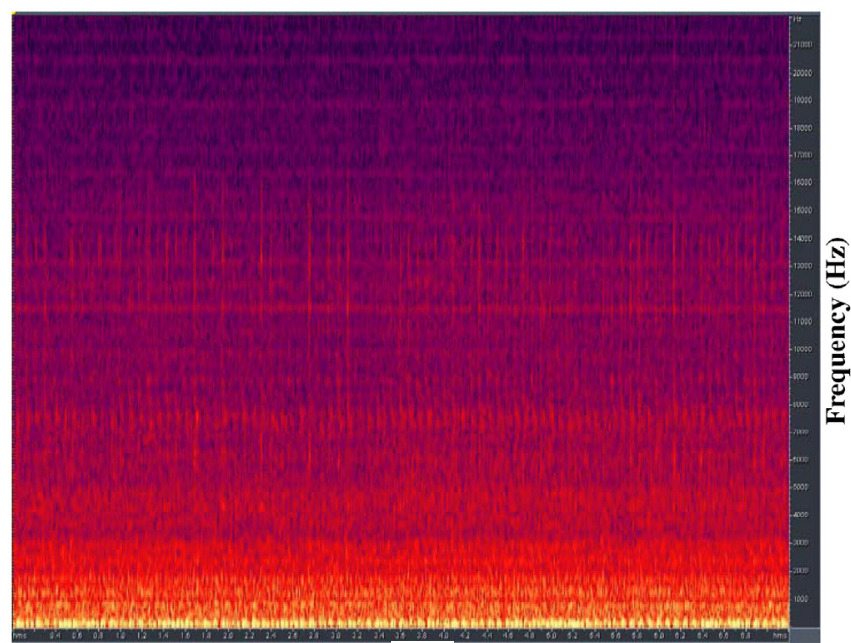

Time (sec)

(c)

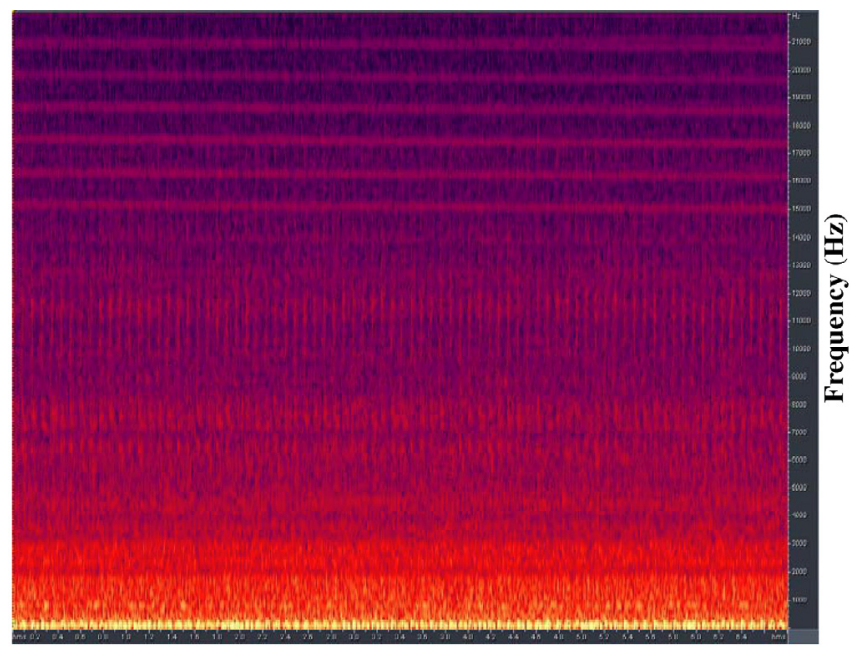

Time (sec)

Fig. (9). The spectrogram of the three kinds of noise. (a) a muffler expansion noise, (b) a one way clutch noise, and (c) a belt damage noise.

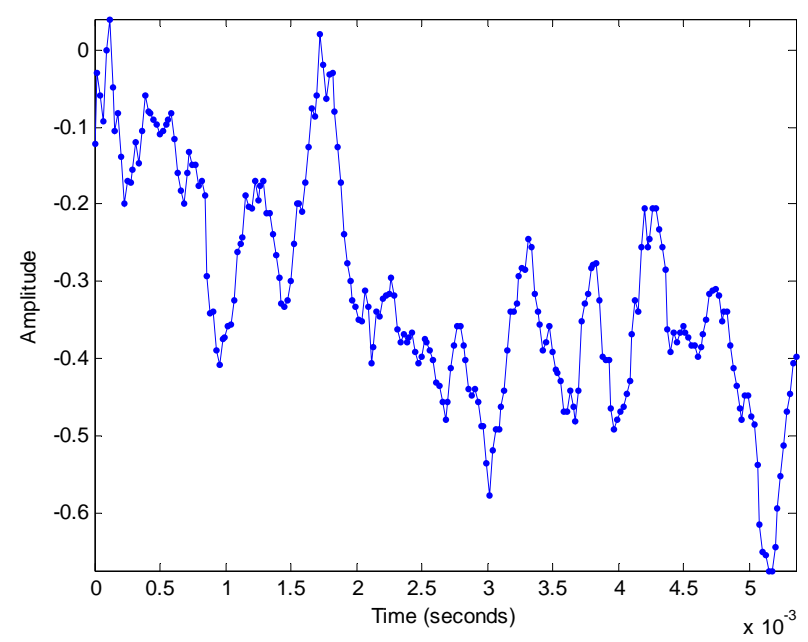

(a)

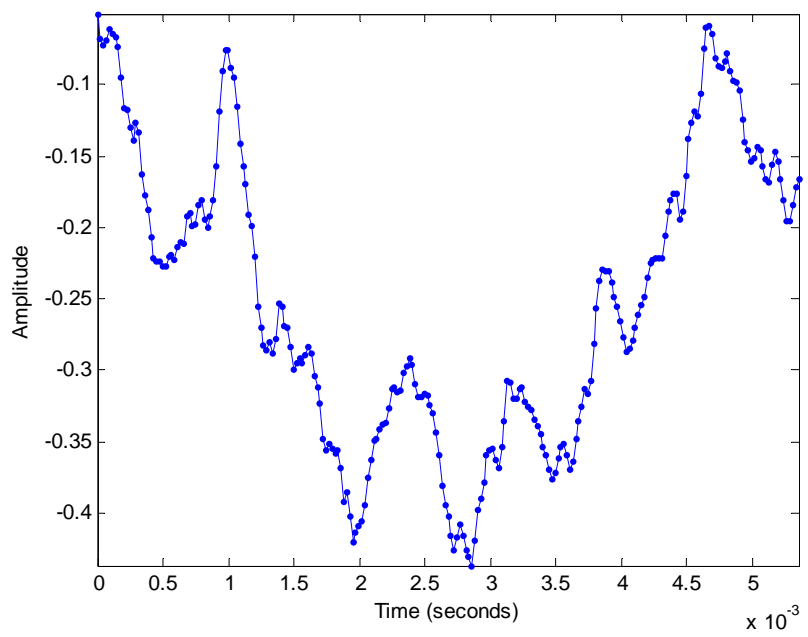

(b)

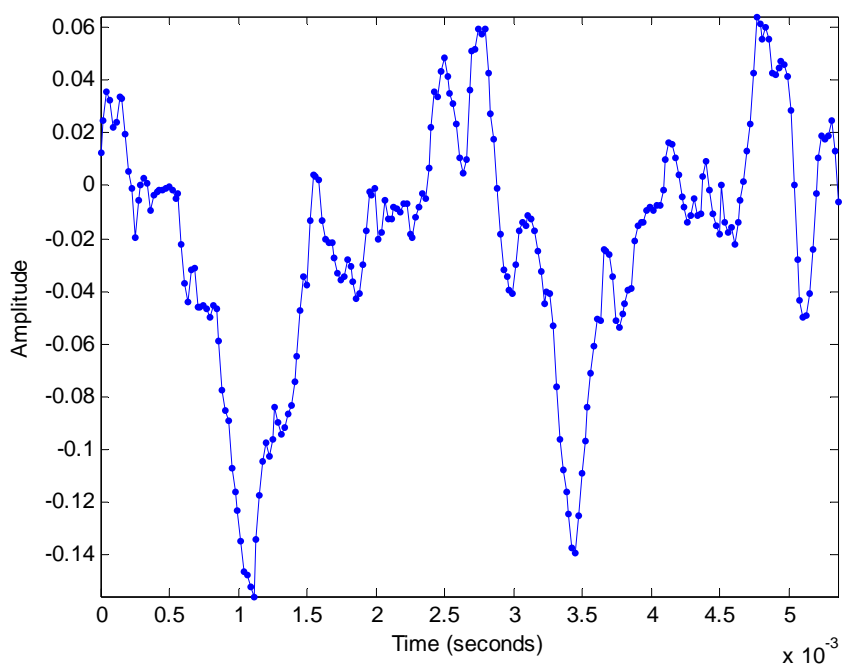

(c)

Fig. (10). The time-domain waveform of three kinds of noise. (a) Muffler expansion noise, (b) one way clutch noise, and (c) belt damage noise. 


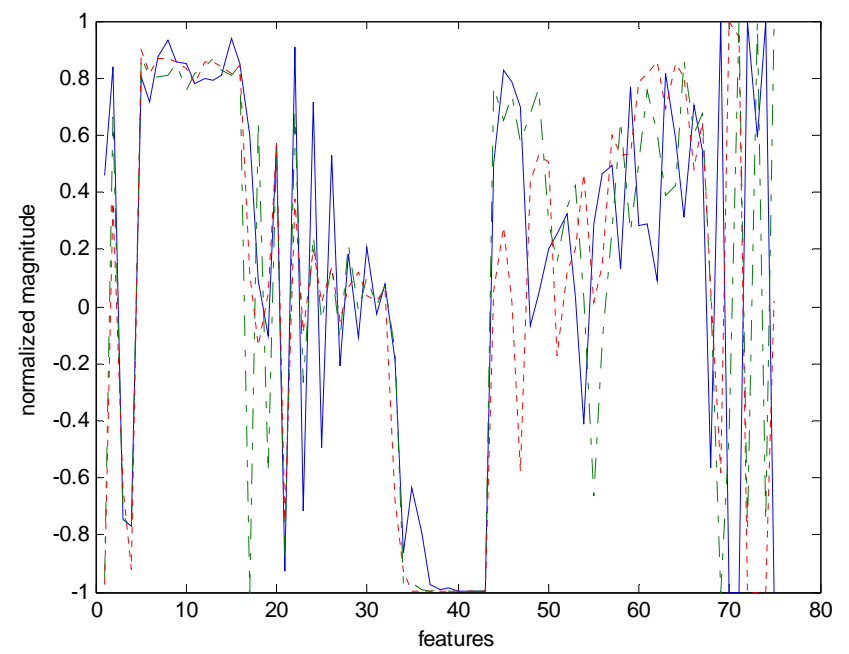

Fig. (11). The features of three kind of noise including a muffler expansion noise (-), a one way clutch noise (-.), and a belt damage noise (:). The abscissa shows the sequence of the features according to APPENDIX.

the ac generator noise and the knocking noise of the engine L-cover seemed to be more difficult to identify than the other fault types, regardless of the classification algorithm used. The successful detection rate of case 3 was also very high (from $96 \%$ of $\mathrm{HMM}$ to $100 \%$ of NNR). A closer inspection of the result reveals that the belt damage was the most difficult fault to identify. In contrast, the engine under normal condition and the air leakage in intake manifold were two easiest fault types for our system. The experimental results suggest that the four classification techniques are effective in identifying fault type correctly using noise data. In particular, FNN has achieved the best performance of fault identification in the test. Introduction of fuzzy logic seemed to have enhanced the performance of plain ANN.

\section{CONCLUSIONS}

A scooter fault diagnostic system that makes use of feature extraction and intelligent classification algorithms has been developed in this study. Nineteen sound features including the MPEG-7 descriptors and several other features in the time and frequency domains are extracted from noise data. The extracted features are normalized prior to classification. Classification algorithms including the NNR, the ANN, the FNN, and the HMM are exploited to identify and classify the scooter noise. The proposed diagnostic system was validated by means of practical noise measurement for various fault types. Experimental results revealed that these four classification techniques had attained high successful detection rate in identifying faults of the scooter. Nevertheless, the identification performance may vary slightly with the algorithm and the type of noise used in the tests. Overall, the classification system based on FNN has achieved the best performance in the test. Introduction of fuzzy logic seemed to have enhanced the performance of plain ANN.

As a limitation of the present research, the proposed technique has been verified only by using internal tests. If external tests are used, where the training data and the testing
Table 1. Identification Results of case 1 Summarized for Four Classification Algorithms. Three Kinds of Scooter Noise Including a Muffler Expansion Noise, a One Way Clutch Noise, and a Belt Damage Noise were Examined

(A)

\begin{tabular}{|c|c|c|c|}
\hline Nearest Neighbor Rule & $\begin{array}{c}\text { Muffler } \\
\text { Expansion }\end{array}$ & $\begin{array}{c}\text { One Way } \\
\text { Clutch }\end{array}$ & $\begin{array}{c}\text { Belt } \\
\text { Damage }\end{array}$ \\
\hline \hline Muffler Expansion & 100 & 0 & 0 \\
\hline One Way Clutch & 0 & 100 & 0 \\
\hline Belt Damage & 0 & 0 & 100 \\
\hline Successful Detection Rate & & $100 \%$ & \\
\hline
\end{tabular}

(B)

\begin{tabular}{|c|c|c|c|}
\hline Artificial Neural Network & $\begin{array}{c}\text { Muffler } \\
\text { Expansion }\end{array}$ & $\begin{array}{c}\text { One Way } \\
\text { Clutch }\end{array}$ & $\begin{array}{c}\text { Belt } \\
\text { Damage }\end{array}$ \\
\hline \hline Muffler Expansion & $\mathbf{1 0 0}$ & 0 & 0 \\
\hline One Way Clutch & $\mathbf{0}$ & 100 & 0 \\
\hline Belt Damage & $\mathbf{0}$ & 0 & 100 \\
\hline Successful Detection Rate & & $100 \%$ & \\
\hline
\end{tabular}

(C)

\begin{tabular}{|c|c|c|c|}
\hline Fuzzy Neural Network & $\begin{array}{c}\text { Muffler } \\
\text { Expansion }\end{array}$ & $\begin{array}{c}\text { One Way } \\
\text { Clutch }\end{array}$ & $\begin{array}{c}\text { Belt } \\
\text { Damage }\end{array}$ \\
\hline \hline Muffler Expansion & 100 & 0 & 0 \\
\hline One Way Clutch & 0 & 100 & 0 \\
\hline Belt Damage & 0 & 0 & 100 \\
\hline Successful Detection Rate & & $100 \%$ & \\
\hline
\end{tabular}

(D)

\begin{tabular}{|c|c|c|c|}
\hline Hidden Markov Model & $\begin{array}{c}\text { Muffler Ex- } \\
\text { pansion }\end{array}$ & $\begin{array}{c}\text { One way } \\
\text { Clutch }\end{array}$ & $\begin{array}{c}\text { Belt } \\
\text { Damage }\end{array}$ \\
\hline \hline Muffler Expansion & 100 & 0 & 0 \\
\hline One Way Clutch & 0 & 100 & 0 \\
\hline Belt Damage & 0 & 0 & 100 \\
\hline Successful Detection Rate & \multicolumn{3}{|c|}{$100 \%$} \\
\hline
\end{tabular}

(E)

\begin{tabular}{|c|c|}
\hline Classification Method & Successful Detection Rate \\
\hline \hline Nearest Neighbor Rule & $100 \%$ \\
\hline Artificial Neural Network & $100 \%$ \\
\hline Fuzzy Neural Network & $100 \%$ \\
\hline Hidden Markov Model & $100 \%$ \\
\hline
\end{tabular}


Table 2. Identification Results of Case 2 Summarized for Four Classification Algorithms. Seven Kinds of Scooter Noise Including the AC Generator Noise, a One Way Clutch Noise, a Noise of the Engine R-Cover, a Knocking Noise of the Engine LCover, a Knocking Noise of the Engine R-Cover, a Knocking Noise of the Engine Back-Cover, and a Knocking Noise of the Engine Top-Cover

(A)

\begin{tabular}{|c|c|c|c|c|c|c|c|}
\hline Nearest Neighbor Rule & AC Generator & $\begin{array}{c}\text { Knocking } \\
\text { Noise of } \\
\text { L-Cover }\end{array}$ & $\begin{array}{c}\text { One-Way } \\
\text { Clutch }\end{array}$ & $\begin{array}{c}\text { Noise- } \\
\text { R-Cover }\end{array}$ & $\begin{array}{c}\text { Knocking } \\
\text { Noise of } \\
\text { R-Cover }\end{array}$ & $\begin{array}{c}\text { Knocking } \\
\text { Noise of } \\
\text { Back-Cover }\end{array}$ & $\begin{array}{c}\text { Knocking } \\
\text { Noise of } \\
\text { Top-Cover }\end{array}$ \\
\hline \hline AC Generator & 76 & 0 & 0 & 24 & 0 & 0 & 0 \\
\hline Knocking Noise of L-Cover & 0 & 89 & 0 & 0 & 11 & 0 \\
\hline Noise-One-Way Clutch & 12 & 0 & 84 & 6 & 0 & 0 & 0 \\
\hline Noise-R-cover & 2 & 0 & 0 & 98 & 0 & 0 & 0 \\
\hline Knocking Noise of R-Cover & 0 & 12 & 0 & 0 & 88 & 0 & 0 \\
\hline Knocking Noise of Back-Cover & 0 & 2 & 0 & 0 & 12 & 0 \\
\hline Knocking Noise of Top-Cover & 0 & 10 & 0 & 0 & 0 & 4 \\
\hline Successful Detection Rate & & & & $86 \%$ & \\
\hline
\end{tabular}

(B)

\begin{tabular}{|c|c|c|c|c|c|c|c|}
\hline Artificial Neural Network & AC Generator & $\begin{array}{c}\text { Knocking } \\
\text { Noise of } \\
\text { L-Cover }\end{array}$ & $\begin{array}{l}\text { One-Way } \\
\text { Clutch }\end{array}$ & $\begin{array}{c}\text { Noise- } \\
\text { R-Cover }\end{array}$ & $\begin{array}{l}\text { Knocking } \\
\text { Noise of } \\
\text { R-Cover }\end{array}$ & $\begin{array}{c}\text { Knocking } \\
\text { Noise of } \\
\text { Back-Cover }\end{array}$ & $\begin{array}{c}\text { Knocking } \\
\text { Noise of } \\
\text { Top-Cover }\end{array}$ \\
\hline AC Generator & 85 & 0 & 0 & 15 & 0 & 0 & 0 \\
\hline Knocking Noise of L-cover & 0 & 84 & 0 & 0 & 16 & 0 & 0 \\
\hline Noise-One-Way Clutch & 0 & 0 & 98 & 2 & 0 & 0 & 0 \\
\hline Noise-R-cover & 0 & 0 & 1 & 99 & 0 & 0 & 0 \\
\hline Knocking Noise of R-Cover & 0 & 10 & 0 & 0 & 90 & 0 & 0 \\
\hline Knocking Noise of Back-Cover & 0 & 2 & 0 & 0 & 0 & 95 & 3 \\
\hline Knocking Noise of Top-Cover & 0 & 0 & 0 & 0 & 0 & 3 & 97 \\
\hline Successful Detection Rate & & & & $92 \%$ & & & \\
\hline
\end{tabular}

(C)

\begin{tabular}{|c|c|c|c|c|c|c|c|}
\hline Fuzzy Neural Network & AC Generator & $\begin{array}{c}\text { Knocking } \\
\text { Noise of } \\
\text { L-Cover }\end{array}$ & $\begin{array}{c}\text { One-Way } \\
\text { Clutch }\end{array}$ & $\begin{array}{c}\text { Noise- } \\
\text { R-Cover }\end{array}$ & $\begin{array}{c}\text { Knocking } \\
\text { Noise of } \\
\text { R-Cover }\end{array}$ & $\begin{array}{c}\text { Knocking } \\
\text { Noise of } \\
\text { Back-Cover }\end{array}$ & $\begin{array}{c}\text { Knocking } \\
\text { Noise of } \\
\text { Top-Cover }\end{array}$ \\
\hline \hline AC Generator & 96 & 0 & 0 & 4 & 0 & 0 & 0 \\
\hline Knocking Noise of L-cover & 0 & 82 & 0 & 0 & 10 & 5 & 0 \\
\hline Noise-One-Way Clutch & 0 & 0 & 99 & 1 & 0 & 0 \\
\hline Noise-R-cover & 0 & 0 & 2 & 98 & 0 & 0 \\
\hline Knocking Noise of R-Cover & 0 & 0 & 0 & 0 & 0 & 0 \\
\hline Knocking Noise of Back-Cover & 0 & 4 & 0 & 0 & 0 & 0 \\
\hline Knocking Noise of Top-Cover & 0 & 0 & 0 & 0 & 1 & 0 \\
\hline Successful Detection Rate & & & & $95 \%$ & 0 \\
\hline
\end{tabular}


(Table 2) contd.....

(D)

\begin{tabular}{|c|c|c|c|c|c|c|c|}
\hline Hidden Markov Model & AC Generator & $\begin{array}{c}\text { Knocking } \\
\text { Noise of } \\
\text { L-cover }\end{array}$ & $\begin{array}{c}\text { One-Way } \\
\text { Clutch }\end{array}$ & $\begin{array}{c}\text { Noise- R- } \\
\text { Cover }\end{array}$ & $\begin{array}{c}\text { Knocking } \\
\text { Noise of } \\
\text { R-cover }\end{array}$ & $\begin{array}{c}\text { Knocking Noise } \\
\text { of Back-Cover }\end{array}$ & $\begin{array}{c}\text { Knocking } \\
\text { Noise of Top- } \\
\text { Cover }\end{array}$ \\
\hline \hline AC Generator & 80 & 0 & 0 & 20 & 0 & 0 & 0 \\
\hline Knocking Noise of L-cover & 0 & 84 & 0 & 0 & 16 & 0 & 0 \\
\hline Noise-One-Way Clutch & 0 & 0 & 90 & 10 & 0 & 0 & 0 \\
\hline Noise-R-cover & 0 & 0 & 10 & 90 & 0 & 0 & 0 \\
\hline Knocking Noise of R-Cover & 0 & 0 & 0 & 0 & 95 & 5 & 0 \\
\hline Knocking Noise of Back-Cover & 0 & 0 & 0 & 0 & 4 & 96 & 0 \\
\hline Knocking Noise of Top-Cover & 0 & 0 & 0 & 0 & 1 & 0 \\
\hline Successful Detection Rate & & & & $90 \%$ & & 0 \\
\hline
\end{tabular}

(E)

\begin{tabular}{|c|c|}
\hline Classification Method & Successful Detection Rate \\
\hline \hline Nearest Neighbor Rule & $86 \%$ \\
\hline Artificial Neural Network & $92 \%$ \\
\hline Fuzzy Neural Network & $95 \%$ \\
\hline Hidden Markov Model & $90 \%$ \\
\hline
\end{tabular}

Table 3. Identification Results of Case 3 Summarized for Four Classification Algorithms. Five Kinds of Scooter Noise Including the Engine Under The Normal Condition, the Noise Due to Intake Manifold Leakage, the Clutch Damage Noise, the Pulley Damage Noise, and the Belt Damage Noise

(A)

\begin{tabular}{|c|c|c|c|c|c|}
\hline Nearest Neighbor Rule & Normal & Air Leakage & Clutch & Pulley & Belt \\
\hline \hline Normal & 100 & 0 & 0 & 0 & 0 \\
\hline Air Leakage & 0 & 100 & 0 & 0 & 0 \\
\hline Clutch & 0 & 0 & 100 & 0 & 0 \\
\hline Pulley & 0 & 0 & 0 & 100 & 0 \\
\hline Belt & 0 & 0 & 0 & 0 & 100 \\
\hline Successful Detection Rate & & \multicolumn{2}{|c|}{$100 \%$} & & \\
\hline
\end{tabular}

(B)

\begin{tabular}{|c|c|c|c|c|c|}
\hline Artificial Neural Network & Normal & Air Leakage & Clutch & Pulley & Belt \\
\hline \hline Normal & 100 & 0 & 0 & 0 & 0 \\
\hline Air Leakage & 0 & 100 & 0 & 0 & 0 \\
\hline Clutch & 0 & 0 & 98 & 2 & 0 \\
\hline Pulley & 0 & 0 & 2 & 98 & 0 \\
\hline Belt & 0 & 0 & 2 & 3 & 95 \\
\hline Successful Detection Rate & \multicolumn{7}{|c|}{$98 \%$} \\
\hline
\end{tabular}

(Table 3) contd.....

(C)

\begin{tabular}{|c|c|c|c|c|c|}
\hline Fuzzy Neural Network & Normal & Air Leakage & Clutch & Pulley & Belt \\
\hline \hline Normal & 100 & 0 & 0 & 0 & 0 \\
\hline Air Leakage & 0 & 100 & 0 & 0 & 0 \\
\hline Clutch & 0 & 0 & 98 & 2 & 0 \\
\hline Pulley & 0 & 0 & 2 & 98 & 0 \\
\hline Belt & 0 & 0 & 3 & 3 & 94 \\
\hline Successful Detection Rate & \multicolumn{7}{|c|}{$98 \%$} \\
\hline
\end{tabular}

(D)

\begin{tabular}{|c|c|c|c|c|c|}
\hline Hidden Markov Model & Normal & Air Leakage & Clutch & Pulley & Belt \\
\hline \hline Normal & 100 & 0 & 0 & 0 & 0 \\
\hline Air Leakage & 0 & 99 & 1 & 0 & 0 \\
\hline Clutch & 0 & 3 & 96 & 1 & 0 \\
\hline Pulley & 0 & 1 & 0 & 97 & 2 \\
\hline Belt & 0 & 4 & 0 & 6 & 90 \\
\hline Successful Detection Rate & \multicolumn{7}{|c|}{$96 \%$} \\
\hline
\end{tabular}

(E)

\begin{tabular}{|c|c|}
\hline Classification Method & Successful Detection Rate \\
\hline \hline Nearest Neighbor Rule & $100 \%$ \\
\hline Artificial Neural Network & $98 \%$ \\
\hline Fuzzy Neural Network & $98 \%$ \\
\hline Hidden Markov Model & $96 \%$ \\
\hline
\end{tabular}




\section{APPENDIX}

Sound features generally fall into. Nineteen features in three categories, spectral features, temporal features and statistical features, are used in this paper. For instance, the spectral features are descriptors calculated using the Short Time Fourier Transform (STFT) or other model-based methods such as Audio Spectrum Centroid, Audio Spectrum Flatness, Linear Predictive Coding (LPC), Mel-scale Frequency Cepstrum Coefficients (MFCC), etc. The temporal features are descriptors calculated using the time-domain signal such as Zero-crossing Rate, Temporal Centroid and Log Attack Time. The statistical features are descriptors calculated based on statistical analysis such as Skewness and Kurtosis. The descriptors from MPEG-7 are marked with “※”.

\begin{tabular}{|c|c|c|c|c|c|}
\hline Spectral Features & Dimension & Sequence & Spectral Features & Dimension & Sequence \\
\hline ※ Audio Spectrum Centroid & 1 & 33 & ※ Harmonic Spectral Centroid & 1 & 69 \\
\hline ※ Audio Spectrum Flatness & 24 & $44 \sim 67$ & ※ Harmonic Spectral Deviation & 1 & 70 \\
\hline ※ Audio Spectrum Envelope & 10 & $33 \sim 43$ & *Harmonic Spectral Spread & 1 & 71 \\
\hline ※ Audio Spectrum Spread & 1 & 68 & ※ Harmonic Spectral Variation & 1 & 72 \\
\hline * Spectral Centroid & 1 & 74 & Sound Pressure Level & 1 & 19 \\
\hline LPC & 13 & $20 \sim 32$ & MFCC & 13 & $4 \sim 16$ \\
\hline Pitch & 1 & 17 & Autocorrelation & 1 & 2 \\
\hline Temporal Features & Dimension & Sequence & Temporal Features & Dimension & Sequence \\
\hline ※ Log Attack Time & 1 & 73 & * Temporal Centroid & 1 & 75 \\
\hline Zero-crossing rate & 1 & 1 & & & \\
\hline Statistical Features & Dimension & Sequence & Statistical Feature & Dimension & Sequence \\
\hline Skewness & 1 & 18 & Kurtosis & 1 & 3 \\
\hline
\end{tabular}

data belong to different set, it is anticipated that not only the detection rate will drop but also the difference in robustness of each classification algorithm against uncertainties and variations of the data may become clear. This conjecture should be examined via external tests in the future research. Extension of the present system to accommodate more fault types by using more features and classification algorithms is currently on the way.

\section{ACKNOWLEDGEMENTS}

The work was supported by the National Science Council in Taiwan, under the project number NSC94-2212-E009018 .

\section{REFERENCES}

[1] Bai MR, Hsiao IL, Tsai HM, Lin CT. Development of an on-line diagnosis system for rotor vibration via model-based intelligent inference. J Acoust Soc Am 2000; 107: 315-323.

[2] Bai MR, Huang JM, Hong MH, Su FC. Fault diagnosis of rotating machinery using an intelligent order tracking system. J Sound Vibrat 2005; 280 (3-5): 699-718.

[3] Jia J, Kong F, Liu Y, et al. Noise diagnosis research based on wavelet packet and fuzzy C-clusters about connecting rod bearing fault. Trans Chin Soc Agric Mach 2005; 36: 87-91.

[4] Wang HM, Chen X, An G, Fan XH. I.C. Engine misfire fault diagnosis based on singular value theory and neural networks. Trans Chin Soc Intern Combust Engines 2003; 21(6): 449-452.

[5] Martinez JM. MPEG-7 Overview (version 9),'ISO/IEC JTC1/ SC29/WG11 N5525, Pattaya, Thailand, March, 2003.

[6] Crysandt H, Wellhausen J. Music classification with MPEG-7," Proc. SPIE Storage and Retrieval for Media Databases, Santa Clara, January, 2003.
[7] Peeters G.A. Large set of audio features for sound description (similarity and classification) in the CUIDADO project, Technical report, IRCAM, Paris, France, 2004.

[8] Peeters G, Rodet X. Automatically Selecting Signal Descriptors for Sound Classification, Proc. International Computer Music Conference, Goteborg, Sweden, September, 2002; pp. 455-458.

[9] Arie A, Livshin, Rodet X. Musical Instrument Identification in Continuous Recordings," Proc. the $7^{\text {th }}$ Int Conf on Digital Audio Effects, Naples, Italy, October, 2004.

[10] Arie A, Livshin, Peeters G, Rodet X. Studies and improvements in automatic classification of musical sound samples," Proc. the International Computer Music Conference, September, 2003.

[11] Zongker D, Jain A. Algorithms for feature selection: An evaluation," Proc. the 13th International Conference on Pattern Recognition, Vienna, Austria, August, 1996.

[12] Cover T, Hart P. Nearest Neighbor pattern classification. IEEE Trans Inform Theor 1967; 13(1): 21-27.

[13] Demuth H, Beale M. Neural Network Toolbox User's Guide, MathWorks, 1998.

[14] Scott P. Music Classification using Neural Networks. Project of EE37B, Stanford university 2001.

[15] Wang CH, Liu HL, Lin CT. Dynamic optimal learning rates of a certain class of fuzzy neural networks and its applications with genetic algorithm. IEEE Trans Syst Man Cybern Part B: Cybernetics 2001;31(3): 467-475.

[16] Li CJ, Lee C, Wang S. Diagnosis and Diagnostic Rule Extraction Using Fuzzy Neural Network, Symposium on Intelligent Systems, International Mechanical Engineering Congress and Exposition, ASME, November 11-16, 2001.

[17] Lin CT, Lee CSJ. Neural Fuzzy Systems, Prentice-Hall, 1996.

[18] Rabiner L. A tutorial on hidden markov models and selected applications in speech recognition. Proc IEEE 1989; 77(2): 257-286.

[19] Chang WS. Development of PSHMM Algorithm and Its Application to Continuous Mandarin Digits Recognition, Master Thesis and Technique Report, Multi-media Information Lab., Institute of Electrical and Control Engineering, National Chiao Tung University, 2001. 
[20] Chai W, Vercoe B. Folk Music Classification Using Hidden Markov Models, Proc. Internation Conference on Artificial Intelligence, Las Vegas, June 2001; pp. 4.3.4/1-5.

[21] Harma A. Linear predictive coding with modified filter structures. IEEE Trans. Speech Audio Proc 2001; 9(8): 769-777.
[22] Logan B. Mel Frequency Cepstral Coefficients for Music Modeling, proceedings of the Int. Symposium on Music Information Retrieval (ISMIR), Plymouth, USA, October 2000.

[23] Oppenheim AV, Schafer RW. Buck JR. Discrete-Time Signal Processing, Prentice Hall, 1999. 